\title{
TURBIDITY STANDARD OF WATER ANALYSIS
}

\author{
By P. V. Wells
}

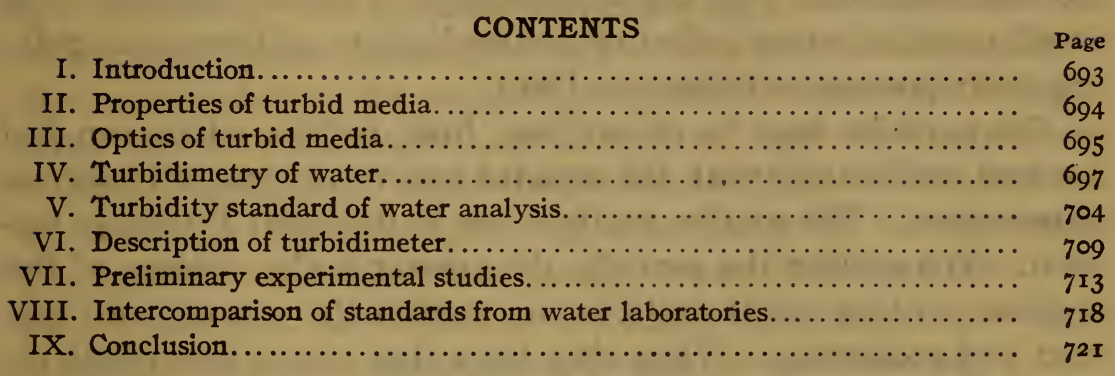

\section{INTRODUCTION}

In practical water analysis the amount of suspended matter has been roughly estimated by the turbidity of the water. In order to render it possible for different laboratories to compare their results the water analysts of the country have adopted a standard of turbidity. This standard, however, has long been considered by them as unsatisfactory, and so it was thought advisable to investigate the subject with the hope of finding some way by which it could be improved. The cooperation of the Bureau was invited by the joint committee on standard methods of the American Public Health Association, the American Chemical Society, and the American Water Works Association.

As a first step in this investigation the methods of measuring turbidity were studied, and a turbidimeter was designed, constructed, and tested. Requests were then sent out to a list of about 30 representative State and municipal water laboratoties for samples of their standard turbidity, and these were intercompared with the turbidimeter. At this point the work was discontinued and parts of the apparatus were used on urgent military problems. The present paper discusses some of the properties of turbid liquids bearing on a standard of turbidity, and describes the methods and results of the intercomparison. 


\section{PROPERTIES OF TURBID LIQUIDS}

The word turbid is derived from the Latin verb turbare, to disturb. The same meaning is attached to the French trouble, and to the German trübe. Hence a turbid medium is one having the mud stirred up. In the scientific sense any medium containing small particles in suspension is turbid. While the original meaning of the word has no direct reference to the eye, turbidity has become associated with the appearance of a turbid medium. The small particles either reflect or scatter light in all directions, making their presence strikingly evident.

The particles may be of any size, from $10^{-3} \mathrm{~cm}$, which can just be seen individually with the unaided eye, to $10^{-8} \mathrm{~cm}$, or molecular dimensions. The smaller particles are in incessant Brownian motion. The smaller the particle, the greater is the violence of this motion, so that small particles are continually coming into contact and coalescing. When they have thus grown into relatively large agglomerates they settle to the bottom of the vessel unless the liquid is stirred mechanically or by convection currents.

When material is dispersed into small particles it presents an enormous surface, so that there may be a relatively large amount of energy represented in the surface layers. Surface phenomena would thus be expected to play an important part in determining the equilibrium. In such cases the conditions of thermodynamic equilibrium in dispersed systems require, according to Tolman, ${ }^{1}$ that the particles shall all be of the same size, and for permanent stability the surface tension between the particles and the liquid must be zero. It is obvious from this that many of the ordinary suspensions of solids in liquids, such as silica and clay suspensions, can not be considered as systems in thermodynamic equilibrium, and in fact it is well known that such suspensions rapidly settle out unless kept well stirred. Very little is known regarding the amount of absorption of the liquid by the dispersed phase, but the relatively large surface would make such an effect important if it existed. In order to have an appreciable concentration represented in the particles enormous numbers must be present, because each one is so very small. Thus, for particles of silica of density 2.6 , and diameter $10^{-5} \mathrm{~cm}$, over $10^{8}$ or 100000000 particles per cubic centimeter must be present to produce a concentration of $\mathrm{IO}^{-8}$, I mg per liter, or as it is commonly expressed, I part per million. It is not surprising that particles crowded together so 
closely coagulate unless forces are constantly at work to keep them separated.

\section{OPTICS OF TURBID MEDIA}

When a turbid medium is illuminated by a beam of light, each particle acts as a secondary source of light. The resultant intensity of the light thus dispersed by the particles in any given direction can be obtained only as the result of an exceedingly complicated calculation, depending on the number, size, shape, and distribution of the particles. Since light is considered to be an electromagnetic wave disturbance, the electrical constants of the particles and of the medium must also be taken into account. From general considerations it is obvious that the phenomena, when the particles are larger than the wave length of the incident light, depend almost entirely upon reflection at the surface of the particles; but when the particles are small compared with the wave length the volume of the disturbing particle is of chief importance, and the phenomenon is then called scattering.

The simplest case is that of a single particle of infinitesimal size compared with the wave length of light. The particle then acts as an electric oscillator, performing forced vibrations in the direction of the impressed force with a certain amplitude, $a$. The oscillator, therefore, sends out scattered waves in all directions, the vibrations being, of course, in every case perpendicular to the direction of the light, since light waves are transverse. But the component of $a$ normal to a line making an angle $\theta$ with the vibration is $a^{\prime}=a \sin \theta$, so that the scattered intensity in this direction, measured by the square of the amplitude, is

$$
I_{\mathrm{s}}=k a^{\prime 2}=k a^{2} \sin ^{2} \theta
$$

Here the incident light is regarded as plane polarized. By ( $\mathrm{I}$ ) the scattered intensity vanishes when $\theta=0$, that is normally to the incident ray, and in the direction of the incident vibration, in agreement with Tyndall's ${ }^{2}$ experiment.

If the light is unpolarized it is more convenient to consider, not the direction of vibration, but the direction of light propagation. If unpolarized light is incident along the axis of $y$, the incident vibration may be regarded as compounded of two vibrations of equal amplitude in the directions of the axes of $x$ and $z$. If the particle is situated at the origin of coordinates, two vibrations of equal amplitude- $a$ along $x$ and $z$-spread out in all

2 J. Tyndall, Cont. to Molecular Physics, Appleton, N. Y., p. 43x, 1897; also Proc. R. S., 108, 1869. 
directions from the origin as from a source. The components of these vibrations perpendicular to a direction $r$, defined by the angles $\alpha, \beta, \gamma$, with the axes of $x, y, z$, are, respectively, $a \sin \alpha$ and $a \sin \gamma$. The resultant intensity $I_{\mathrm{s}}$, of the scattered light along $r$, is

$$
I_{\mathrm{s}}=k a^{2}\left(\sin ^{2} \alpha+\sin ^{2} \gamma\right)
$$

but from geometry $\cos ^{2} \alpha+\cos ^{2} \beta+\cos ^{2} \gamma=\mathrm{I}$, and hence

$$
I_{\mathrm{s}}=k a^{2}\left(\mathrm{I}+\cos ^{2} \beta\right)
$$

This gives the variation of the scattered intensity with the angle between the directions of the incident and scattered light. The intensity is a maximum in the direction of the incident light, decreasing to one-half normally, and zero in the opposite direction.

The amplitude of the vibration in the scattered light, on either the elastic solid theory or the electromagnetic theory of light, is proportional to the volume, $V$, of the small disturbing particle. At a distance $r$ from the particle the amplitude must be inversely proportional to $r$, so that in order to be dimensionally correct, the ratio of the amplitudes $a$ of the scattered light and $a_{0}$ of the incident light of wave length $\lambda$ must be

$$
\frac{a}{a_{\mathrm{o}}}=k \frac{V}{\lambda^{2} r}
$$

These simple considerations may help to explain Rayleigh's expression for the intensity $I_{\mathrm{s}}$ of the light scattered from $N$ particles each of volume $V$, the incident intensity being $I_{0}$ and the wave length $\lambda$. This is ${ }^{3}$

$$
\frac{I_{\mathrm{s}}}{I_{\mathrm{o}}}=\frac{n^{\prime 2}-n^{2}}{n^{2}} \frac{N V^{2}}{\lambda^{4} r^{2}}\left(\mathrm{I}+\cos ^{2} \beta\right)
$$

Here $n$ is the refractive index of the medium, $n^{\prime}$ that of the particles. The particles are supposed to be contained in such a small volume that the distance $r$ and the angle $\beta$ between the scattered and incident beams are the same for all the particles. For particles of different size, all small compared with the wave length, a summation must be made, requiring the size distribution of the particles. When the particles are not small compared with the wave length, terms of higher order must be included, and again the expression becomes complicated.

No account is taken in (5) of secondary scattering. All of the light scattered by the particles is supposed to reach the eye with-

${ }^{3}$ Lord Rayleigh, Sci. Papers, 1, pp. 87-rro, Camb. Univ. Press; 1899. 
out loss. When the medium is densely filled with particles, this factor may become of first importance. The fractional decrease of the intensity $I$ in traversing a thickness, $d x$, of the turbid medium is

$$
\frac{d I}{I}=-\frac{h}{\lambda^{4}} d x
$$

where $h$ is a constant independent of $\lambda$.

Integrating

$$
I_{\mathbf{x}}=I_{\mathrm{o}} e^{-\frac{h x}{\lambda^{4}}}
$$

where $I_{0}$ is the intensity of the light when $x=0$ and $I_{x}$ is the intensity after traversing a thickness $x$.

The most striking characteristic of equations (5) and (7) is the occurrence of the factor $I / \lambda^{4}$, indicating that the scattered light increases rapidly as the wave length decreases. The scattered light is, therefore, much bluer than the incident light, while the blue is correspondingly absent in the transmitted portion, which contains a relatively large fraction of the red light. This was used by Rayleigh to explain the blue color of the sky, as well as the red colors of the sunset. The blue color may be used as a test of the size of the particles of any turbid medium. Thus the fine blue smoke from the end of a cigar is an indication that the smoke particles are much smaller than the wave length of light. Tyndall's test of complete polarization at right angles to the incident beam is still more sensitive.

Strutt " has recently observed the light scattered by the molecules of gases, and finds that it is not completely polarized, as demanded by the simple theory. He considers this to be due to the departure of the molecules from spherical symmetry. It is not necessary here to go into the large amount of research, both theoretical and experimental, that has been done on the optics of turbid media. Enough has already been given to show that these phenomena are very complicated.

\section{TURBIDIMETRY OF WATER}

Natural waters are almost always found to have foreign matter in suspension. The suspended matter is usually silt and clay abraded from the soil over which the water flows, and prevented from settling by convection currents. Organic material is also found, sometimes in large quantities. This is usually of microscopic size, and is different from the mineral ingredients both in 
density and color. It is obviously impossible to completely describe the important properties of suspended matter composed of particles of widely different sizes and materials by means of a single quantity. Turbidity is, therefore, usually defined as the mass of suspended matter per unit volume. In absolute cgs units turbidity is expressed in grams per cubic centimeter, while in technical water analysis it is usually expressed in parts (by weight) per million. The density and size of the suspended particles are thus ignored.

When the particles are large it is not difficult to remove them from the liquid by filtering, washing, and drying, and then the mass can be determined directly by weighing. When the particles are smaller than the pores of the filter they must be increased in size by some method of coagulation. If there are no nonvolatile substances in solution, it is not necessary to filter, for the volatile constituents can be removed by evaporating the medium to dryness directly. But these methods are limited by the precision of the balance.

It is possible to detect, optically, the presence of suspended matter when the gravimetric method fails, completely. In fact, it is difficult to obtain liquids which are optically clear, except in a few special cases. This is particularly true of water. Optical methods of measuring turbidity are, therefore, especially valuable in the analysis of waters containing very small traces of suspended matter.

The optical methods of measuring turbidity fall into two classes, (I) those in which the measured quantity is the thickness of the turbid medium required to cause some standard object to disappear, and (2) those in which the quantity measured is the intensity of the light, either reflected or scattered by the turbid medium. These may be briefly called (I) disappearance and (2) intensity methods, respectively.

Disappearance methods have arisen from the demands of measurements in the field. Thus the platinum-wire method, now used as the standard for water analysis, as originally designed by Hazen, ${ }^{5}$ was simply a pin stuck into a piece of wood and lowered into sewage until it vanished. In its present form it is designed for the field assay of water. ${ }^{6}$ Similarly for over a hundred years the oceanographer has lowered circular disks of canvas into the sea to

5 Hazen, A., Filtration of Public Water Supplies, 3d ed., Wiley \& Sons, New York.

${ }^{6}$ See Leighton, M. O., Field Assay of Water, U. S. Geol. Surv., Water Supply and Irrigation Paper No. 151, Washington, D. C.; 1905. 
measure the depth at which they disappeared. This method is an obvious outgrowth of the simple observation of objects beneath the surface of the sea, which in clear tropical waters are sometimes visible to a depth of over 80 fathoms. The determining factors in field methods are simplicity, speed, and ease of carrying, not precision and reliability. But even in field methods the desire for simplicity decreases with familiarity, and the faults become increasingly evident, so that the tendency is ever toward increasing precision, which usually involves more complicated apparatus. This is illustrated by the development of the candle and electric turbidimeters for field work.

The earliest form of laboratory apparatus using the disappearance principle was the diaphanometer designed by Horning ${ }^{7}$ in I876. This instrument was improved by Parmelee and Ellms, ${ }^{8}$ Jackson, Leighton ${ }^{9}$, and Weaver ${ }^{10}$, and is used in the chemical laboratory for rapid work. It usually consists of a cylindrical glass Nessler tube, sealed and polished flat on the bottom. The turbid liquid is poured into this until the test object just disappears from view. Jackson and Leighton used for a test object a slit, $0.5 \mathrm{~mm}$. wide, in the form of a cross illuminated from below by an electric lamp, but I am informed by Mr. Weaver that the incandescent filament of a carbon lamp gives a sharper and more reproducible end point. The depth of the liquid is then measured. In order to exclude the stray light, the turbidity tube is inclosed in a metal casing, which in the Smith ${ }^{11}$ form is perforated with a slit parallel to its axis and graduated so that the depth can be read directly in parts per million. The electric bulb is also inclosed in a light-tight metal chamber.

The theory of the disappearance method is very complicated, but it is found empirically that for particles of a given size the concentration, $C$, is related to the depth $z$ over small ranges by an expression of the form

$$
C=k z^{-\alpha}
$$

where $k$ and $\alpha$ are constants.

This equation will be considered later in connection with the turbidity standard of water analysis. From the optics of the question it is evident that regardless of loss of definition the test

${ }^{7}$ Horning, G., Engineering Naw.s, 35-36, p. 219 ; 1896.

${ }^{8}$ Parmelee, C. L., and Ellms, J. S., Technology Quarterly, 12, pp. 145-164; 1899.

9 Loc. cit. See note 6.

${ }^{10}$ R. S. McBride and E. R. Weaver, Bureau of Standards Tech. Paper No. 20, p. 36; r9r3.

11 O. M. Smith, U. S. Patent Nos. 1, 232, 989, 10, July, 1917 . $150053^{\circ}-20-2$ 
object disappears when its brightness is just equal to the brightness of the turbid medium. Now its brightness depends upon ( $x$ ) its illumination, (2) its reflection coefficient, and (3) the fraction of this reflected light transmitted through the medium to the eye. Similarly the brightness of the turbid medium depends upon the integrated effect of a host of particles, each of whose differential contributions to the brightness depends upon (I) its illumination, (2) its coefficient of reflection, or of scattering if the particle is smaller than the wave length of the light, and (3) the loss in transmission to the eye. The particles situated between the test object and the eye contribute light which is superposed on the image of the object itself. In addition to these brightness effects the definition of the image of the test object is impaired by the blur circles of images of particles out of focus, and by diffraction of the rays from the test object around the edges of the particles. Disappearance methods thus involve an estimation by the eye of brightness contrast and definition. The factors producing this criterion all vary as we pass from one disappearance method to another, so that each must be considered separately.

In the laboratory form of turbidimeter described above, the controlling factors are simplest and most nearly constant. This is the reason for its precision and reliability. The test object is an incandescent carbon filament. The fraction of the light reaching the eye is given by a decreasing exponential function as in equation (7). The illumination of the suspended particles comes from the slit which is used as the test object, or from other particles by secondary scattering. The brightness of the turbid medium is thus an integrated effect of all the particles. Since the brightness, both of the filament and of the medium, is proportional to the intensity of the source, changes in the voltage of the lamp only influence the color effect. For particles small enough to obey Rayleigh's law the source appears redder than the medium, but for large particles this is not troublesome as the reflected light is practically the same for all colors.

Intensity methods have been developed to meet the needs of the laboratory in measuring small turbidities. Here simplicity is not the prime requisite, and the attempt is made to obtain the utmost precision and reliability. The growth of such instruments is quite recent, with the exception of the nephelometer of Richards, ${ }^{12}$ which appeared in its first form in 1894 . It is designed 
for the photometric comparison of two turbid liquids of nearly equal physical properties with high precision. In research on the optics of turbid media many experimenters have used apparatus for measuring the intensity of light scattered by a turbid medium. ${ }^{13}$

In 1914 Mecklenburg and Valentiner ${ }^{14}$ described a tyndallmeter by which the light scattered by a turbid medium perpendicularly to the incident beam can be measured. Recently Tolman ${ }^{15}$ has designed an instrument for measuring the turbidity of smokes as well as of liquids.

Intensity methods possess great advantages over disappearance methods in sensitiveness. They are thus particularly adapted to the measurement of exceedingly small traces of turbidity. The sensitiveness is due to the fact that the eye never views the source of illumination directly, but only the light scattered by the particles, against a perfectly black background. It is what is known to the microscopist as dark-ground illumination, the principle underlying the design of the ultramicroscope. A source of great intensity is used to illuminate the particles, and the brightness of the turbid medium in a given direction is measured photometrically. For simplicity the direction usually chosen is that perpendicular to the incident beam. If the medium is perfectly clear, the field is absolutely black, in spite of the fact that a powerful beam of light is passing through the medium.

It is obvious that the slightest amount of stray light may upset the measurement completely, so that great care must be taken that the background be absolutely black, and that any glass surfaces in the field be either perfectly polished, free from scratches, or be in such a position that the powerful beam does not shine directly or indirectly upon them. Scrupulous cleanliness is also required in the cell containing the turbid medium to insure that the turbidity is due, not to the cell, but to the medium itself.

The brightness of the turbid medium is a complicated function of the concentration of the dispersed phase. For particles of one substance and one size, small compared with the wave length of the light employed, the brightness $B$ of a thin section of the medium would be expected to be, from Rayleigh's expression (5)

$$
\frac{B}{B_{o}}=\frac{k d^{3} C x}{\lambda^{4}}
$$

${ }^{13}$ See for instance papers by Rayleigh (187r), Abney (r886), Hurion (189r), Lampa (18)I), Barnes (1902), Ehrenhaft (1903), Müller (1907), Bence (1909), Herxheimer (I912), and Keen and Porter (1914).

$14 \mathrm{~W}$. Mecklenburg and S. Valentiner Zeitschrift für Instrumentenkunde; July, 1914.

15 R. C. Tolman, Jour. Am. Chem. Soc., 41. 
where $B_{o}$ is the brightness of a comparison field illuminated by the same source, $C$ the concentration (mass per unit volume), $d$ the uniform diameter of the particles, $x$ the thickness of the section in the direction viewed, $\lambda$ the wave length of light employed, and $k$ a constant. On the other hand, for particles larger than the wave length of light the brightness would be expected to be proportional to the total surface of the particles, and to be dependent on the color only as the reflection coefficient varies with $\lambda$. Thus for large particles

$$
\frac{B}{B_{o}}=\frac{k C x}{d}
$$

In ordinary suspensions particles of many different sizes are usually found. The brightness of such a turbid medium depends upon the size distribution of the particles and may be much more complicated than either (9) or (Io). Moreover, the effect of secondary scattering and reflection, and of absorption, have been entirely ignored in both (9) and (Io). For particies of certain substances and of sizes not negligibly small compared with the wave length, the light scattered shows marked color effects, usually ascribed to resonance phenomena ${ }^{16}$. It is evident from these considerations that the brightness of a turbid medium can not be taken as a measure of the concentration except in very special cases, for it varies rapidly with the size of the suspended particles.

The occurrence of many variables in the expression for the brightness of the turbid medium does not impair its use for the comparison of turbid media similar in all respects and only slightly different in concentration. It was for just such purposes that Richards and Wells used the nephelometer. Two samples of known concentration are successively compared with the unknown, the concentration of which is obtained by interpolation. Over such small ranges the other variables may be regarded as sensibly constant.

Both classes of optical methods, (I) the disappearance and (2) the intensity methods, are dependent upon the sensitiveness of the eye. Both involve the photometric comparison of the brightnesses of two adjacent objects, but this is complicated in disappearance methods by the estimation of definition. Under the best conditions, namely, (I) sufficient illumination, (2) exact juxtaposition of the two fields, and (3) perfect color match, the photometer is 
capable of a precision of better than one-half of i per cent. Very slight departures from ideal conditions reduce the precision rapidly to not better than I per cent.

In turbidimetry the photometric conditions are often far from ideal. With media of slight turbidity the illumination is below that at which the eye is most sensitive, with disappearance methods and also with intensity methods unless a powerful beam of light is used. The selective action of the turbid medium produces a color which varies with the sample measured. Of course, this does not affect the nephelometer, which merely compares two similar samples, but tyndallmeters suffer from this variable color difference between the photometric fields. If monochromatic light is used the loss in intensity seriously affects the sensitiveness.

In passing it may be useful to note that for the purposes of chemical analysis the turbidimeter is of wide utility, wherever it is desired to measure quickly fine precipitates which do not coalesce and settle rapidly. For high turbidities the disappearance method is very serviceable, because of its simplicity and precision. For extremely small turbidities it is necessary to use an intensity method.

In routine water analysis the turbidity is ordinarily estimated by comparison of the sample with the standard under similar conditions. The instructions given in Standard Methods of Water Analysis, 1917, page 5, are as follows: "Comparison with the standards shall be made by viewing both standard and sample sidewise toward the light by looking at some object and noting the distinctness with which the margins of the object can be seen." The method is thus essentially a distinctness test, similar in principle to the disappearance methods. The sample and the standard must be in similar bottles or tubes and they must be under the same illumination.

In testing this method the cylindrical shape of the bottles and tubes was found unsatisfactory for distinctness judgments. Cells with parallel flat faces were preferable. But if care was taken to have uniform illumination, and the samples were viewed against a black screen with the eye properly protected from the direct light, satisfactory results were obtained without using any distinctness test. Turbidity differences of 15 per cent were almost certain of detection, but some errors were made in comparing turbidities differing by ro per cent. For the purposes intended the method seems to be quite satisfactory. Its accuracy can not be greater 
than that of the standards used for comparison. For turbidities differing much in color the distinctness test is preferable and the precision is much less.

\section{TURBIDITY STANDARD OF WATER ANALYSIS}

As the result of a comparative study of the methods in use for measuring the turbidity of water, Whipple and Jackson ${ }^{17}$ recommended as the standard of turbidity water containing a definite amount of powdered silica obtained from diatomaceous earth. This recommendation has been followed and now constitutes the turbidity standard of water analysis. The complete specifications for this standard are given in Standard Methods of Water Analysis, third edition, published by the American Public Health Association, Boston, I9I 7, pages 4 to 9. The first paragraph is as follows:

The standard of turbidity shall be that adopted by the United States Geological Survey, namely, a water which contains roo parts per million of silica in such a state of fineness that a bright platinum wire $\mathrm{I} \mathrm{mm}$ in diameter can just be seen when the center of the wire is roo $\mathrm{mm}$ below the surface of the water and the eye of the observer is 1.2 meters above the wire, the observation being made in the middle of the day, in the open air, but not in sunlight, and in a vessel so large that the sides do not shut out the light so as to influence the results. The turbidity of such water is arbitrarily fixed at roo parts per million.

Comparison standards are obtained by dilution of a stock suspension containing 1000 parts per I 000 ooo by weight of silica. Comparison with these standards is made by noting the distinctness of objects seen through the samples. The platinum-wire methods for regulating the fineness depends upon the fact that for the particles used the vanishing depth increases with the size of particle. The particles required to give the proper reading are of the same size as clay, which is the most important ingredient of turbid waters from the standpoint of filtration.

Any standard may be judged from three points of view, namely, (I) accuracy, (2) convenience, and (3) range of application. The primary requisite of a standard, no matter what its convenience or range of application, is sufficient accuracy for its reliable use. It must be constant, permanent, and reproducible. Given these, the less important qualities of convenience and range of application must be considered.

The present standard of turbidity for water analysis is prepared according to specification in each local laboratory, introducing an unnecessary lack of uniformity. The regulation of the fineness 
by the platinum-wire method is relatively difficult of performance, and depends upon meterological conditions which are not constant. Laboratories are, therefore, deterred from reproducing the stock suspension frequently; but unfortunately the silica suspension is not in stable equilibrium, the particles coagulate slowly, so that the standards decrease in turbidity on standing. It is appropriate and simple in use, so that as regards convenience there is little to be desired. The range of application of the turbidity standard is, unfortunately, limited, but this is unavoidable, as it is due to the complex nature of dispersoids in general, and particularly to the wide variety of turbid waters which must be utilized for water supplies. When it is considered that moving water carries in suspension objects of weight increasing with the sixth power of the velocity of the current, it is not surprising that natural waters contain particles of widely different sizes. But the same law is just as efficient in allowing the larger particles to settle out in reservoirs where the water is still, leaving in suspension only some of the finer silt, the clay, and organic material. This varies widely in color, according to the nature of the suspended matter, and colored samples are not easily compared with the bluish-white standard silica suspensions, even when the criterion used is the distinctness of objects seen through the sample. This limitation has caused the rejection of the standard in some localities.

The use of the platinum-wire method as a means of regulating the fineness of the standard is the least satisfactory part of the specification, from the standpoint of accuracy. The rate of change of the vanishing depth with the size of particle is rather small, as is the precision of the reading itself, so that the standard may vary considerably from uniformity in size of particle. The law of the instrument is rather complicated and seems to be entirely empirical. The relation between the concentration, $C$, and the vanishing depth, $z$, is best represented by the equation

$$
C=k z^{-\alpha}
$$

but this holds only for small ranges. If this be fitted to the smoothed data for the turbidity rod of $1902,{ }^{18}$ the formula (8) is found to hold within I per cent over the ranges and with the constants given in Table $\mathrm{I}$. The concentration is in parts per million by weight, the depth in millimeters. 
TABLE 1

\begin{tabular}{|c|c|c|}
\hline $\begin{array}{c}\text { Valid range } \\
\text { of } C \\
\text { (parts per } \\
1000000)\end{array}$ & $\alpha$ & $\begin{array}{c}\text { Constants } \\
k\end{array}$ \\
\hline $7-100$ & 1.11 & $1.67 \times 10^{4}$ \\
$100-200$ & 1.25 & $3.95 \times 10^{4}$ \\
$200-400$ & 1.44 & $6.67 \times 10^{4}$ \\
$400-800$ & 1.67 & $1.56 \times 10^{5}$ \\
$800-3000$ & 2.01 & $4.42 \times 10^{5}$ \\
\hline
\end{tabular}

But if the formula be fitted to the points ro and 3000 parts per million, the constants are $\alpha=\mathrm{I} .36$ and $k=8.5 \mathrm{I} \times \mathrm{IO}^{4}$, and while it fits at the ends, it is in error 66 per cent at roo parts per million. The simpler formula

$$
C=\frac{7850}{z-9}
$$

fitted in the same manner is in error by only 14 per cent at roo parts per million. This formula is less satisfactory theoretically, for it indicates that $C$ is infinite when $z=9$, instead of the proper value of $C$, about 4300 . Moreover, if fitted to the ranges 7 to roo and 800 to 3000 parts per million, deviations from the observed values of 5 and 12 per cent are found while equation (8) fits to I per cent over these ranges. Neither formula is good enough for practical use over the whole range. For turbidities below roo parts per million the concentration is roughly proportional inversely to the vanishing depth, while for turbidities above 800 parts per million it is nearly inversely proportional to the square of the vanishing depth. Between roo and 800 parts per million there is a transition in $a$, the exponent of $z$. A knowledge of the size of particles used in obtaining the data upon which this calibration is based might be of interest in this connection.

A few experiments were made in order to become familiar with the platinum-wire method. A piece of platinum wire, $\mathrm{I} \mathrm{mm}$ in diameter (within less than I per cent), and $25 \mathrm{~mm}$ long was sealed into the side of a glass tube within which was inserted a millimeter scale. The silica suspension was placed in a liter graduate $6 \mathrm{~cm}$ in diameter and $40 \mathrm{~cm}$ deep. This was immersed in a large glass vat, $30 \mathrm{~cm}$ in diameter, $60 \mathrm{~cm}$ deep, until the level in the vat was $3 \mathrm{~mm}$ below that of the liquid in the graduate. The turbidity rod was clamped in a telescope stand and adjusted vertically by the eye. Thus the observer could lower the rod by means of a rack and pinion smoothly at any desired slowness, 
and could hold it indefinitely at a given point without muscular effort. The turbidity vat was placed directly below a skylight in an attic room which gave diffuse light simulating a cloud. The turbidity of the liquid in the vat was roughly equal to that in the liter graduate.

The results of the first day with four observers, all experienced in physical measurements, are summarized in Table 2. In the first column is given the serial number of the observer, in the second the number of depth observations taken by him, in the third the mean of his vanishing depth readings, $z$; in the fourth the average deviation of his individual observations from his mean, and in the last column the deviations of the means of the single observers from the weighted mean of all the means.

TABLE 2

\begin{tabular}{|c|c|c|c|c|}
\hline Jan. 9, 1914 (observer) & $\begin{array}{l}\text { Number } \\
\text { of } \\
\text { observa- } \\
\text { tions }\end{array}$ & $\begin{array}{l}\text { Mean } \\
\text { vanishing } \\
\text { depth } \\
z(\mathrm{~mm})\end{array}$ & $\begin{array}{l}\text { Average } \\
\text { deviation, } \\
\text { single ob- } \\
\text { servations }\end{array}$ & $\begin{array}{c}\text { Deviations } \\
\text { from } \\
\text { weighted } \\
\text { mean }\end{array}$ \\
\hline 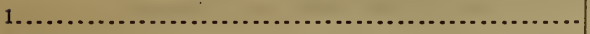 & 13 & 46.22 & 1.23 & 1.17 \\
\hline $2 \ldots \ldots$ & 14 & 46.13 & 1.32 & 1.08 \\
\hline 3........ & 22 & 43.66 & 1.75 & 1.39 \\
\hline $4 \ldots \ldots \ldots . . . . .$. & 7 & 45.21 & 1.39 & .16 \\
\hline 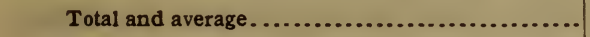 & 56 & 45.05 & 1.42 & .95 \\
\hline
\end{tabular}

NotE. -Weighted mean $45.05 \pm 0.95=2 . x$ per cent; maximum diffcrence $=2.56=5.7$ per cent.

TABLE 3

\begin{tabular}{|c|c|c|c|c|}
\hline Jan. 10. 1914 (observer) & $\begin{array}{l}\text { Number } \\
\text { of } \\
\text { observa- } \\
\text { tions }\end{array}$ & $\begin{array}{l}\text { Mean } \\
\text { vanishing } \\
\text { depih }\end{array}$ & $\begin{array}{l}\text { Average } \\
\text { deviation, } \\
\text { single ob- } \\
\text { servations }\end{array}$ & $\begin{array}{l}\text { Deviations } \\
\text { from } \\
\text { weighted } \\
\text { mean }\end{array}$ \\
\hline $1 \ldots \ldots \ldots \ldots$ & 9 & 46.03 & 1.77 & 2.37 \\
\hline $1 \ldots \ldots \ldots \ldots$ & 6 & 46.49 & 1.07 & 1.91 \\
\hline $1 \ldots \ldots \ldots . . . .$. & 2 & 51.60 & 1.10 & 3.20 \\
\hline $1 \ldots \ldots \ldots . . . . .$. & 4 & 53. 58 & 1.25 & 5.18 \\
\hline $4 \ldots \ldots \ldots \ldots \ldots$ & 14 & 47.68 & 2.03 & .73 \\
\hline 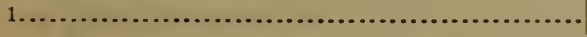 & 5 & $51.56^{\circ}$ & .80 & 3.16 \\
\hline Total and average. . & 40 & 48.40 & 1.34 & 2.76 \\
\hline
\end{tabular}

Note. - Weighted mean $48.40 \pm 2.76=5.7$ per cent: maximum difference $=7.55=$ i 5.6 per cent.

These results show no systematic differences between the four observers, who consistently reproduced their single observations to 3 per cent, and agreed in their mean results to 2 per cent, the maximum difference being less than 6 per cent. The sun appeared bright through a uniform mist, and remained the 
same from II.3O a. m. to $2.30 \mathrm{p}$. m. during the observations. There was indication that the longer the time occupied in making an observation, the greater the vanishing depth obtained.

The results of the next day, given in Table 3 , were not so favorable.

An increase in the reading seemed to occur with practice; but the results with an observer (5) skilled in estimating the brightness of faint stars showed the most disconcerting disagreement. These are given in Table 4 .

TABLE 4

\begin{tabular}{|c|c|c|c|c|}
\hline Jan. 13, 1914 (observer) & $\begin{array}{l}\text { Number } \\
\text { of obser- } \\
\text { vations }\end{array}$ & $\begin{array}{c}\text { Mean } \\
\text { vanishing } \\
\text { depth }\end{array}$ & $\begin{array}{l}\text { Average } \\
\text { deviation, } \\
\text { single ob- } \\
\text { servation }\end{array}$ & $\begin{array}{c}\text { Deviation } \\
\text { irom } \\
\text { weighted } \\
\text { mean }\end{array}$ \\
\hline $1 . \ldots \ldots \ldots \ldots$ & 6 & 53.98 & 1.0 & 7.87 \\
\hline $1 \ldots \ldots \ldots \ldots \ldots$ & 5 & 56.46 & 1.6 & 5.39 \\
\hline $5 \ldots \ldots \ldots \ldots \ldots$ & 6 & 74.22 & 6.2 & 12.37 \\
\hline Total and average.. & 17 & 61.55 & 2.9 & 8.54 \\
\hline
\end{tabular}

NoTE. - Weighted mean $6 \mathrm{r} .55 \pm 8.54=13.9 \mathrm{pcr}$ cent; maximum difference $=20.24=32.7$ per cent.

The observer (5) took great care, despite the discrepancy in his single observations. There is no reason to suppose that his interpretation of the criterion might not be duplicated by other observers preparing the standard according to specifications.

The experiments were performed merely to become familiar with the procedure of the platinum-wire method. A critical study of the method would require an extended experimental investigation. Nevertheless, the results agree with those of other observers.

The choice of platinum wire I mm thick as the test object, the eye being placed at a distance of $1.2 \mathrm{~m}$, produces an image so small that it can hardly be resolved by the eye. The limit of resolution is usually taken as I minute of arc. The bright line on the wire due to reflection probably does not subtend at the eye an arc of more than 3 minutes, but the effect of irradiation tends to obliterate a fine, dark line on a brighter background. Thus, a strip of black paper I mm wide on a sheet of white paper disappeared at about I $_{5} \mathrm{~m}$, while a strip of the same white paper I $\mathrm{mm}$ wide on a sheet of the same black paper disappeared at nearly $30 \mathrm{~m}$ under the same conditions. This effect, combined with the light superposed on the image from the turbid medium between the wire and the eye, sufficiently explain the failure of 
the wire to reappear as a dark line in a brighter field when lowered below the critical depth, regardless of any loss of definition. But whether the criterion of disappearance is a matching of brightness, is simply a loss of definition, or is a combination of both remains an open question.

The turbidity standard of water analysis is regarded as unsatisfactory by many water analysts. Some consider it inaccurate, others inconvenient, and others find it inapplicable to particular water supplies. The question of accuracy obviously should be considered first. Our preliminary study of the problem indicates (I) that the theory is very complicated, (2) that the standards would not be expected to be permanent for any length of time, and (3) that the preparation of the standard by means of the platinum-wire method at each laboratory would be expected to lead to considerable lack of uniformity. An intercomparison of the laboratory standards was, therefore, undertaken to determine the accuracy of the present standards in actual use.

\section{DESCRIPTION OF TURBIDIMETER}

The superiority of intensity methods of measuring turbidity, both from the theoretical aspect and in the criterion which forms the basis of the measurement, led to the adoption of this principle in the design of a turbidimeter for the experimental investigation. After a few experiments and a preliminary instrument ${ }^{19}$ had been studied it became evident that no one instrument is satisfactory for use over a wide range of turbidities. For turbidities above $10-^{5} \mathrm{~g}$ per cubic centimeter (ro parts per I 000 o0o by weight), sensitiveness is not the primary consideration, and such requisites as simplicity, convenience, and size may be included. Below $10-{ }^{5}$ ( 10 parts per I 000 o00) strict attention to sensitiveness must be given. The final design ${ }^{20}$ adopted is shown in the photographs, Figs. I and 2. The instrument was constructed in the Bureau instrument shop by $\mathrm{H}$. C. Wunder.

The essential purposes of the design-great sensitiveness, combined with as wide a range as possible-required a variable thickness of cell and the measurement of the light scattered by the sample at a small angle from the incident beam. It was also advisable to have this angle variable, in order to be able to compare the scattered light from the sample with the reflected

19 This instrument was described in a paper presented to the Physical Society, Apr. 24, 19r4. See Phys. Rev., 1, p. 396; 1914.

${ }^{20}$ A much simpler instrument would have sufficed for the present investigation, but further research was contemplated which led to this design. 
light from an opaque surface. The instrument was not inclosed, in order to give greater freedom for experiments, and also because stray light could thus be better controlled.

The source of light used was a ro8-watt concentrated filament locomotive-headlight lamp of high efficiency, taking 5.5 volts. The rays from the lamp were made parallel by a cemented triplet lens $5 \mathrm{~cm}$ in diameter and $\mathrm{r} 2 \mathrm{~cm}$ in focal length. The parallel beam thus produced passed through a plate-glass reflector and was incident at a slight angle, usually $20^{\circ}$ from the normal to the face of the cell containing the turbid sample. The intensity of the scattered light was measured with a Martens polarization photometer placed in such a position that the axial ray of one field passed through the middle of the cell containing the sample and normally to its face. In the comparison field of the photometer was a diffusing screen of ground opal glass. This was illuminated by the reflected portion of the parallel beam from the plate-glass reflector, after passing through total reflecting prisms to provide for the rotation of the photometer about a vertical axis.

The cell containing the turbid sample was made so that it could be chemically cleaned. A cylindrical hole about $5 \mathrm{~cm}$ in diameter was bored through a sheet of plate glass of the desired thickness. Thin sheets of plate glass were held by compression against the bored plate, forming a cell of glass. The pressure was produced by two plates of brass with four long bolts. The glass pieces could thus be removed, taken apart, and soaked in hot cleaning solution, washed, dried, and put together with the cell walls, and especially the faces, spotlessly clean. A series of these cells was made of thicknesses varying from 2 to $38 \mathrm{~mm}$ to determine the variation in the reading with the thickness. The lamp house and collimating lens, mounted on a single sliding base, could be moved away from the photometer to make room for long cells for gases. The cells were mounted on a plate supported by three adjusting screws, and were placed against an adjustable stop so that they could be replaced quickly and accurately in the same position.

The lamp was mounted on an adjustable base so that new lamps could be easily inserted in precisely the proper position, namely, so that the filament was in the optical axis of the system. It was inclosed in a ventilated light-tight lamp house. The stray light from the opening toward the lens was absorbed by sheets of black cardboard properly placed.

The photometer was mounted on an arm, so that it could be rotated about a vertical axis. In this way the optical axis of the 
Scientific Papers of the Bureau of Standards, Vol. 15

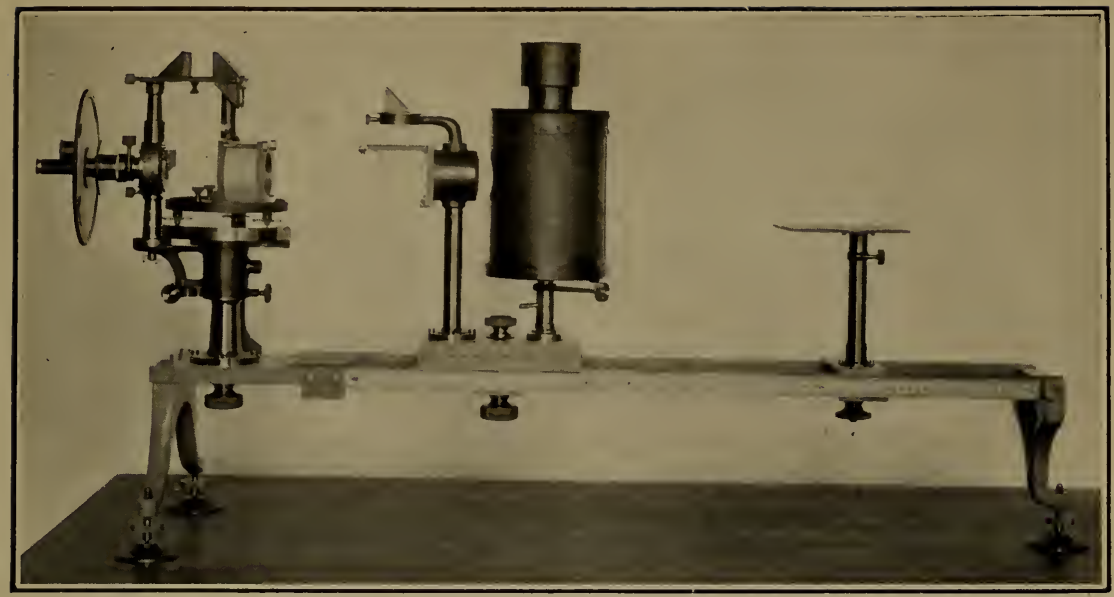

FIG. I.-Instrument as used for measuring intensity of scattered light at angle of $20^{\circ}$

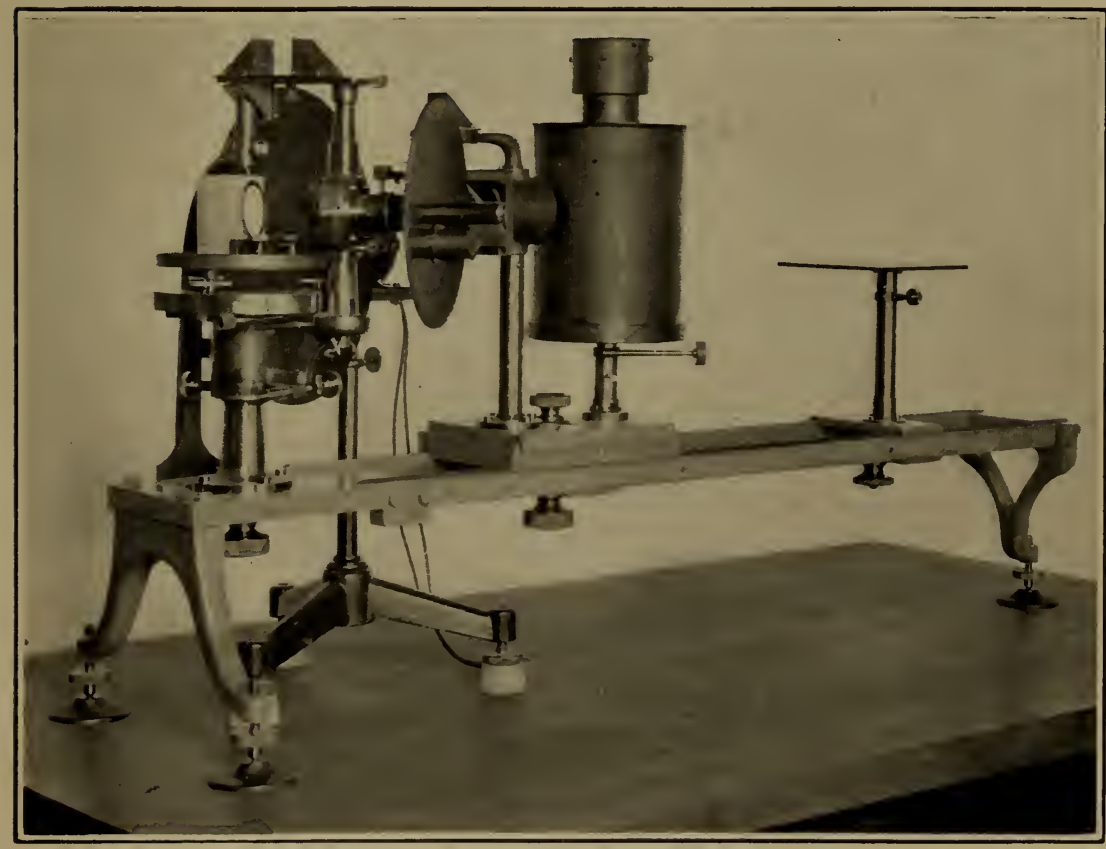

FIG. 2.-Instrument as used for measuring light reflected from a standard magnesia surface 
photometer could be set at any desired angle with the axis of the parallel incident beam. An inclosed circle, graduated in degrees, was provided for measuring this angle. Fig. I shows the instrument adjusted for measuring the light scattered at an angle of $20^{\circ}$ by the turbid sample in a cell $38 \mathrm{~mm}$ thick, the face of which was normal to the optical axis of the photometer. Fig. 2, on the other hand, shows the instrument adjusted for measuring the light reflected by a standard magnesia surface normally, the parallel beam being incident at an angle of $45^{\circ}$. The surface here is also normal to the photometer axis. A sectored disk is also shown in this figure, which was used for reducing the intensity of the incident beam to bring the brightness of the magnesia surface within the range of the photometer.

The Martens polarization photometer is an instrument of high precision, which on account of its convenient size, and design, is very useful in the optical laboratory. It is not as popular in technical photometry, because there the polarization of the light is not of interest, and this instrument measures the component in one plane. The two fields measured by the photometer are each about $5^{\circ}$, with their axes about $10^{\circ}$ apart. These fields are brought into precise juxtaposition by means of a biprism and their brightness adjusted to equality by means of a combination of a Wollaston prism as a polarizer and a Nicol prism as an analyzer. A circle graduated in degrees measures the angle between the principal planes of the prisms. The law of the instrument is

$$
R=k \tan ^{2} \omega
$$

where $R=A_{1} / B$ is the ratio of the component of the brightness of one field $\left(A_{1}\right)$ in the vertical plane to the component of the brightness of the other field $(B)$ in the horizontal plane. The angle $\omega$ is that between the principal planes, and is given by $\omega=\alpha+\delta$, where $\alpha$ is the observed angle and $\delta$ is the displacement of the index on the scale. When properly adjusted $\delta$ should be zero. The constant $k$ should equal unity if the transmission of the optical system of the photometer is the same for both beams. It was found that $k$ was equal to I within o.I per cent. The zero reading $\delta$ could be reduced to o. ${ }^{\circ}$ (less than 0.2 per cent), but it was tedious to adjust it more closely with the coarse adjustment provided. It was eliminated very easily by taking the mean of the readings in two adjacent quadrants. A careful study of the instrument has shown it to have a precision under the best conditions of better than 0.5 per cent. 
Of course, this is not required in turbidimetry, where the precision is limited by other than photometric factors.

The photometer readings are most accurate when comparing two fields of equal brightness. When the brightness ratio exceeds 300 or is less than 0.03 , the stray light in the photometer appreciably affects the reading. The range of the photometer was then extended by using a sectored disk. This was made adjustable to any transmission up to 35 per cent. The circle was graduated to read directly in per cent transmission, and could be set easily with a precision equal to its accuracy. The edges of the sectors were made of steel, accurately ground straight, and adjustable to fit each other exactly when shut. The index on the divided circle was set on zero when the edges were in contact, eliminating any correction. The whole disk was made symmetrical about the axis to avoid any strains due to centrifugal action. It was mounted directly on the shaft of a o.oI-horsepower i Io-volt direct-current series motor, and rotated with a speed which averaged about $1670 \mathrm{rpm}$. There were thus about 56 flashes per second, which is much above flicker speed. Yet at perfect match it was often possible to detect a slight flickering just at the dividing line between the two fields, which disappeared when the fields were not matched. The sectored disk was carefully investigated on the precision circular dividing engine of the Bureau, especially with regard to eccentricity of the graduated circle and the axis of rotation, and also with regard to the accuracy of the edges. In no case was an error in one sector of more than five minutes of arc detected, and the average was about two minutes. But $5^{\prime}=0.083^{\circ}$, so that the minimum angle which could be used with a precision of I per cent was $8.3^{\circ}$, which corresponded to a transmission of 4.6 per cent. The sectored disk was thus accurate much beyond what was required in this work when used at transmissions above 5 per cent. Actually it was never used below 8 per cent.

The most important optical consideration in any turbidimeter is stray light. This introduces constant errors which may become very serious with small turbidities. Stray light may come from three main sources, (I) reflections from the walls of the instrument, (2) reflections from the cell walls, and (3) imperfections on the cell faces. Reflections from the walls of the instrument were completely eliminated by having no walls visible in the field of the photometer. Black silk velvet was placed at a distance opposite the photometer field, giving an absolutely black field. 
Diaphragms were used to cut off the incident light from the cell walls. The scattered light from the turbid medium was reflected by the walls back into the medium again, causing a slight increase in the illumination of the particles. This must have been a second order effect, and was probably negligible. The most important stray light was that coming from scratches and particles on the cell faces. When the incident beam illuminated these faces a constant amount of stray light was produced directly in the field of the instrument and changed the zero reading. It was corrected by taking the zero reading with optically clear water. Doubly distilled water was used, which always contained plenty of turbidity, and this masked the zero reading due to the cell faces themselves. With air in the cell, the conditions were not exactly comparable, but this gave a fairly good idea of the amount of stray light from the faces themselves. The zero reading was reduced to a minimum by using carefully polished, scratch-free, optically clean glass faces.

\section{PRELIMINARY EXPERIMENTAL STUDIES}

In order to test the turbidimeter, it was necessary to have standards which remained constant. Three such standards were used, designated $U_{10}, U_{11}$, and $U_{12}$. $U_{10}$ was a piece of opal glass faced with clear glass, to which it was fused. The clear-glass faces were carefully polished optically flat by the Bureau optician, J. Clacey. In order to reduce the brightness of this; a plate of neutral-tint smoke glass was used. The whole was properly mounted so that it could be placed in a given position and adjusted in the same way as the turbidity cells. $U_{11}$ and $U_{12}$ were also pieces of opal glass, but instead of clear glass both faces were carefully ground with fine emery powder, producing uniform diffusing white-glass surfaces. The polished surfaces of $U_{10}$ permitted of thorough cleaning and precise adjustment of its faces, while the diffusing surfaces of $U_{11}$ and $U_{12}$ were less sensitive to changes of direction and parallelism of the incident beam. They were also found not to change when the surfaces were washed with soap and water.

It is not necessary here to describe in detail the tests made with these constant standards to determine the causes of the variations in the readings, the reproducibility of the adjustments and of various parts of the apparatus, and the final methods adopted for keeping the instrument in adjustment. The most 
important causes of variation were those due to the source of light and those due to cleaning the various optical surfaces. A telescope focussed for parallel rays was used for adjusting the filament of the source to the principal focus of the collimating lens. The optical axes of the system were adjusted by reflection methods. It was found that no single adjustment determined the precision of the instrument. Table 5 below gives all the readings obtained during a period of two months upon the three standards $U_{10}, U_{11}$, and $U_{12}$ to discover a secular change in the standards or the instrument. The readings are referred to their mean values for comparison. Each reading is the mean of ro observations. During the interval all the adjustments were remade, the optical surfaces cleaned, and a new lamp inserted and readjusted.

TABLE 5.-Constancy of Instrument

\begin{tabular}{|c|c|c|c|}
\hline Data & $U_{10}$ & $\tau_{11}$ & $U_{12}$ \\
\hline \multirow{8}{*}{ Readings } & 1.07 & 1.03 & 1.04 \\
\hline & .95 & .97 & .98 \\
\hline & .96 & .97 & .96 \\
\hline & .96 & .99 & .99 \\
\hline & .98 & .97 & .98 \\
\hline & 1.07 & 1.07 & 1.05 \\
\hline & 1.05 & 1.04 & 1.03 \\
\hline & .96 & .96 & .96 \\
\hline \multirow{4}{*}{ 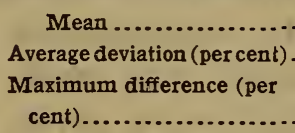 } & 1.00 & 1.00 & 1.00 \\
\hline & 4.8 & 3.5 & 3.3 \\
\hline & & & \\
\hline & 12.0 & 11.0 & 10.0 \\
\hline
\end{tabular}

The variations are due obviously to erratic changes in adjustment. No secular change in the standards is noticeable. The variations in $U_{11}$ and $U_{12}$ are less than those in $U_{10}$, probably because they were less sensitive to adjustment due to their diffusing surfaces. The standard $U_{10}$ was used over a much longer period-seven months, including the readings in Table 5 . The average deviations of a single reading from the mean of all (22 readings) was 4.3 per cent, and Table 5 itself includes the highest and lowest readings of the whole series. In fact, if the readings of the first five months only were considered, the average deviation was 2.2 per cent, and the maximum difference is 9 per cent. When the instrument was kept fixed in adjustment the readings were more nearly constant. 
A few experiments were made upon silica suspensions to determine the errors involved with liquid standards. The magnitude of the zero reading due to stray light from the cell faces was found to vary considerably, unless great care was taken to wash the cells as well as the cell faces perfectly clean. With proper precautions the zero reading could be kept below that due to a turbidity of $1 \mathrm{O}^{-8}$ (0.01 parts per $\mathrm{I}$ 000 000 ), and with ordinary technique it was below $\mathrm{IO}^{-7}$ (0.I parts per I 000000 ), so that without correction it would introduce an error of less than I per cent in turbidities greater than $\mathrm{IO}^{-5}$ (IO parts per I 000000 ).

A considerable error may be introduced in the process of filling and washing the turbidity cells, to avoid which a definite procedure must be followed. Washing the cells three times with doubly distilled water, and then once with the sample, was found to give results better than 2 per cent in most cases.

When much time is consumed in making the observations, as is usually necessary in photometric work, an error is introduced due to the settling of coarser particles of the suspension. A rough time run was taken to determine the rate of settling with a standard suspension of $1 \mathrm{O}^{-4}$ (100 parts per million) concentration. The fractional rate of settling is given by $s=\mathrm{I}-S$, where $S$ is the fraction remaining in suspension after unit time. Thus, if $R_{0}$ is the original reading, and $R$ the reading after $t$ minutes,

$$
R=R_{0} S t
$$

The results are given in Table 6 .

TABLE 6.-Rate of Settling

\begin{tabular}{|c|c|c|c|c|c|}
\hline Time (p. m.) & $\underset{R}{\text { Reading }}$ & $\log R$ & $\log S$ & $S$ & $\begin{array}{c}s \\
\text { per cent } \\
\text { per minute }\end{array}$ \\
\hline $1.40 .$. & 12.95 & 1.1123 & 0.9953 & 0.989 & 11 \\
\hline $1.45 .$. & 12.27 & 1.0888 & & & \\
\hline $1.50 \ldots$ & 11.99 & 1.0788 & . 9980 & .995 & .5 \\
\hline $3.00 \ldots$ & 9.05 & .9566 & .9983 & .996 & .4 \\
\hline
\end{tabular}

It is seen that for the first five minutes the reading decreases at the rate of about I per cent per minute, after which time a steady rate of about one-half of $\mathrm{I}$ per cent per minute is reached. The time element is uncertain because the readings themselves require about five minutes for a series of ro photometric observations. The results show that care must always be taken to shake the standard suspension well immediately before the observations. 
Here a difficulty is encountered, for shaking produces bubbles which increase the reading.

The most important factor in the calibration of any turbidimeter is the determination of the relation between the reading and the concentration, for every type of suspension which is to be used. The simplest method of obtaining the concentration as a function of the reading is to dilute the suspension by known amounts and obtain the readings for the successive dilutions. The resulting function may be called the law of dilution. It is assumed in this procedure that the constitution of the suspension does not change on dilution, but the particles may break up or in some way change in size, involving in itself a change of reading.

TABLE 7.-Law of Dilution

\begin{tabular}{|c|c|c|c|}
\hline \multicolumn{2}{|c|}{$\underset{C}{\text { Concentration }}$} & \multirow{2}{*}{$\underset{R}{\text { Reading }}$} & \multirow{2}{*}{$\begin{array}{l}\text { Obs.-Calc } \\
\frac{\Delta C}{C}, \text { per cen }\end{array}$} \\
\hline $\begin{array}{l}\text { Grams per } \\
\text { cubic } \\
\text { centimeter }\end{array}$ & $\begin{array}{l}\text { Parts par } \\
\text { million }\end{array}$ & & \\
\hline $5.00 \times 10^{-5}$ & 50.0 & 103.0 & +11.9 \\
\hline 2.92 & 29.2 & 74.1 & +3.5 \\
\hline 1.70 & 17.0 & 49.0 & -2.8 \\
\hline $9.90 \times 10^{-6}$ & 9.90 & 31.5 & -5.7 \\
\hline 5.76 & 5.76 & 19.4 & -5.4 \\
\hline 3.36 & 3.36 & 11.9 & -3.8 \\
\hline 1.96 & 1.96 & 7.18 & 0.0 \\
\hline 1.14 & 1.14 & 4.37 & +2.1 \\
\hline $6.64 \times 10^{-7}$ & .664 & 2.63 & +6.9 \\
\hline 3.87 & .387 & 1.69 & +3.0 \\
\hline 2.26 & .226 & 1.01 & +7.6 \\
\hline 1.31 & .131 & .636 & +5.7 \\
\hline $7.66 \times 10^{-8}$ & .0766 & .394 & +7.4 \\
\hline 4.46 & .0446 & .283 & -10.4 \\
\hline 2.60 & . 0260 & .194 & -22.5 \\
\hline Mean. & & & 6.6 \\
\hline
\end{tabular}

A given thickness of cell is appropriate only for a definite range of turbidities, and for each cell thickness the constants in the law of dilution are different. The $38 \mathrm{~mm}$ cell was found to be suitable for silica suspensions of concentrations ranging from $5 \times 10^{-5}$ (50 parts per I 000000 ) to $3 \times 10^{-8}$ (0.03 parts per I 000000 ), and over this range the law of dilution was obtained. A suspension containing $5 \times 10^{-5}$ (50 parts per million) of silica was first read. Twenty-five cubic centimeters were removed with a pipette and replaced by doubly distilled water, and as the 
total volume was $60 \mathrm{~cm}^{3}$, the concentration was reduced by a factor of seven-twelfths at each dilution. The data obtained in one run are given in Table 7 .

The observed reading is corrected for the displacement of the photometer scale $\left(\delta=+0.14^{\circ}\right)$ and for the zero reading with doubly distilled water, which averaged $1.7 \times 10^{-8}$ (0.01 7 parts

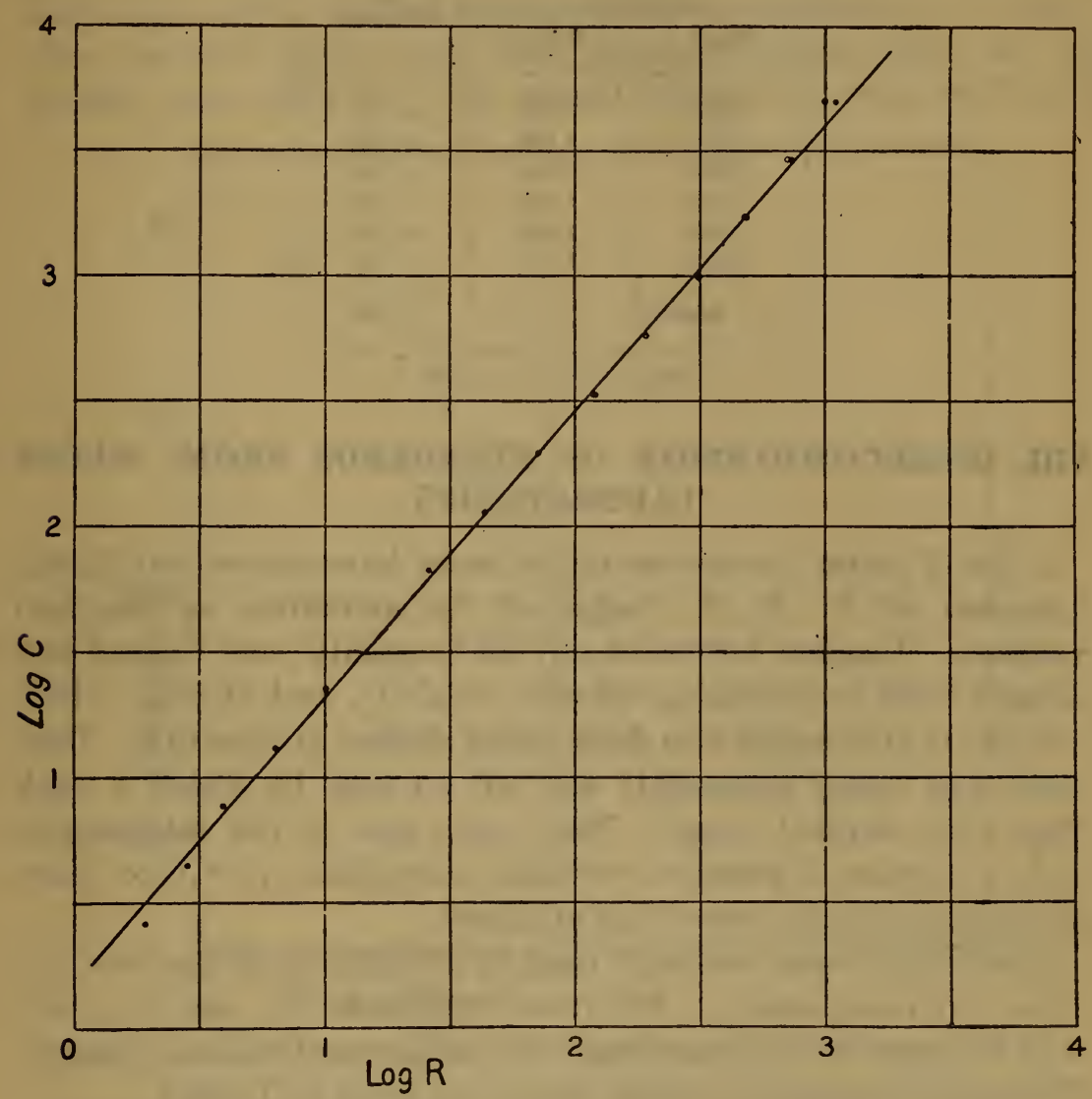

Fig. 3.- Shows relation between concentration $(C)$ and turbidimeter readings $(R)$

per million). The data are fitted fairly well by the formula

$$
C=k R^{\alpha}
$$

in which $C$ is the concentration, $R$ the corrected turbidimeter reading, and $k$ and $\alpha$ constants. The data are plotted logarithmically in Fig. 3, giving the value $\alpha=1.194$, the observed values of $C$ differing from the calculated on the average by 6.6 per cent. In reality the law is much more complicated, as is shown by the systematic deviations in the last column of Table 7 . 
The results of a trial run made on the previous day check fairly well, as is shown in Table 8 , the average difference between the readings being 2.2 per cent. They are also plotted in Fig. 3 .

TABLE 8.-Reproducibility of Dilution

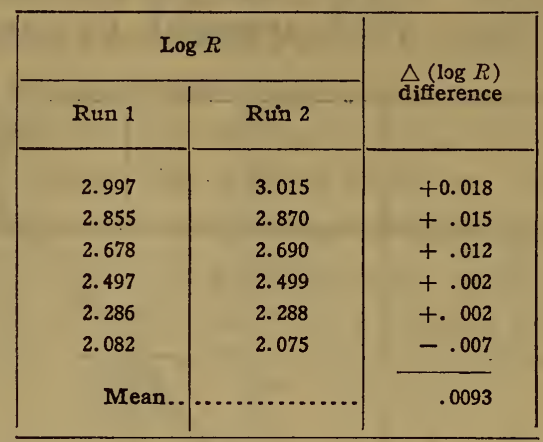

Mean $\Delta R=2.2$ per cent.

\section{INTERCOMPARISON OF STANDARDS FROM WATER LABORATORIES}

A list of about 30 representative water laboratories was kindly furnished by Dr. E. B. Phelps, of the committee on standard methods. Reagent bottles of $500 \mathrm{~cm}^{3}$ capacity were washed and cleaned with hot cleaning solution $\left(\mathrm{K}_{2} \mathrm{Cr}_{2} \mathrm{O}_{7}\right.$ and $\left.\mathrm{H}_{2} \mathrm{SO}_{4}\right)$, which was left in the bottles two days, being shaken at intervals. They were then rinsed thoroughly and left to soak for about a week filled with distilled water. These were sent to the laboratories and 22 samples of standard turbidity containing $\mathrm{IO}^{-4}$ (IOO parts per million) of silica were thus obtained.

The turbidimeter was kept fixed in adjustment throughout the whole intercomparison. Reference standards $U_{11}$ and $U_{12}$ were read at intervals to insure that the adjustment did not change. The readings referred to their means are given in Table 9.

TABLE 9.-Constancy During Intercomparison

\begin{tabular}{|c|c|c|}
\hline Data & $U_{11}$ & $U_{12}$ \\
\cline { 1 - 2 } & 1.00 & 1.00 \\
Readings ..................... & 1.03 & 1.00 \\
& .97 & 1.02 \\
1.00 & .98 \\
Mean ......................... & $\overline{1.00}$ & 1.00 \\
Average deviation (per cent)... & 1.5 & 1.0 \\
Maximum difference (per cent). & 6.0 & 4.0 \\
\hline
\end{tabular}


The variations are evidently accidental, and not due to changes in adjustment.

A definite procedure was followed in reading the turbidity standards. In each case the cell was washed three times with doubly distilled water and emptied with a vacuum siphon with a fine tip to suck the liquid from the corners. The third washing was read, giving the readings in Table ro marked D. D. $\mathrm{H}_{2} \mathrm{O}$. The standard sample was then carefully shaken and the cell washed once with it. The second filling was read, with and

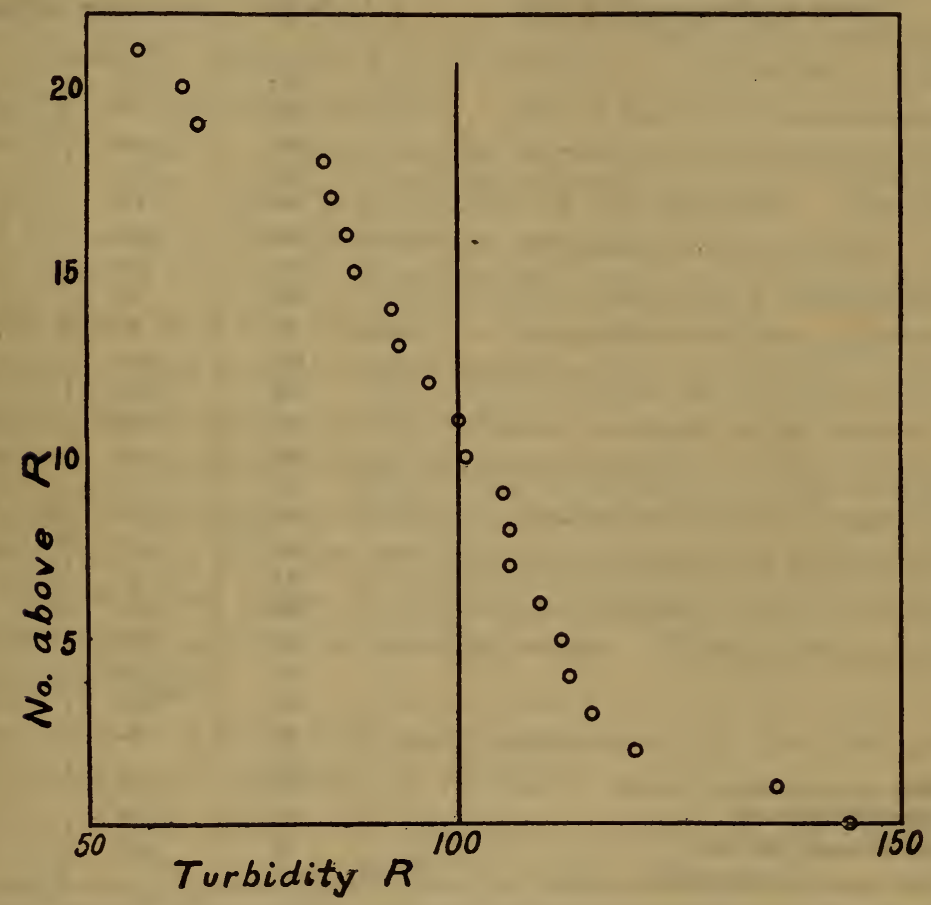

FIG. 4.-Shows the lack of uniformity in standards of turbidity used in practice

without a green filter over the eyepiece to eliminate small color differences. Five samples were remeasured several times and were found to check in every case except one to better than 3 per cent, averaging I.3 per cent. One reading was higher than the mean of three by ro per cent, probably due to an error in washing and filling, as the sample was low and the reading was taken immediately after a high sample. Readings were taken with and without a green filter over the eyepiece, five observations each, and the means are given in the second and third columns of Table 10 . The readings referred to the mean of all 
which is fixed at Ioo, as the standards were supposed to be 100 parts per million by weight. In the last column are given the differences between the readings without and with the green filter. The average difference is 3 per cent, the maximum ro per cent. These differences may be due to color variations among the samples, but the average reading with the green filter was I0.64, with no filter 10.56, a difference of 0.8 per cent.

TABLE 10.-Intercomparison of Standards

\begin{tabular}{|c|c|c|c|}
\hline Doubly-distilled water (D. D. $\mathrm{H}_{2} \mathrm{O}$ ) & No filter & Green filter & Difference \\
\hline $0.9 .$. & 107 & 106 & +1 \\
\hline $.3 \ldots$ & 100 & 97 & +3 \\
\hline $.3 .$. & 86 & 89 & -3 \\
\hline $.9 \ldots$ & 118 & 118 & 0 \\
\hline $.6 \ldots$ & 85 & 83 & +2 \\
\hline $1.1 \ldots .$. & 115 & 119 & -4 \\
\hline $.9 . . .$. & 114 & 108 & +6 \\
\hline $.8 \ldots$ & 82 & 81 & +1 \\
\hline $.7 \ldots$ & 83 & 85 & -2 \\
\hline $.5 \ldots$ & 153 & 162 & -9 \\
\hline $.8 \ldots \ldots \ldots$ & 143 & 146 & -4 \\
\hline $.5 . \ldots \ldots$. & 57 & 56 & +1 \\
\hline $1.1 \ldots$ & 107 & 97 & +10 \\
\hline $1.1 \ldots$ & 91 & 89 & +2 \\
\hline $.5 \ldots$ & 111 & 112 & -1 \\
\hline $.6 \ldots .$. & 124 & 126 & -2 \\
\hline $.7 \ldots .$. & 65 & 64 & +1 \\
\hline $.8 \ldots$ & 106 & 113 & -7 \\
\hline $.8 \ldots .$. & 92 & 90 & +2 \\
\hline $.6 \ldots .$. & 63 & 62 & +1 \\
\hline $.7 \ldots$. & 101 & 103 & -2 \\
\hline $.8 \ldots \ldots \ldots$ & 96 & 94 & +2 \\
\hline Mean .. & 100 & 100 & $\ldots$. \\
\hline Average deviation (per cent)........ & 18 & 19 & 3 \\
\hline Maximum difference (per cent)........... & 96 & 107 & 10 \\
\hline
\end{tabular}

The readings without filter are presented graphically in Fig. 4, which is a Galton ogive curve, or " direct plot." The abcissas are the readings $R$, ordinates, the number of readings above $R$; each reading thus being given an equal ordinate spacing, and arranged in order of magnitude.

The results show a rather serious lack of uniformity in the turbidity standard actually in use. It was at first suspected that this was due to variations in the methods of preparation, such as the use of diatomaceous earth, local clays, the candle turbidimeter, etc., but a study of the results indicates that they are due to chance irregularities, not to systematic differences of 
method. The lowest three and the highest two readings fall apart from the rest, and are perhaps exceptional, but omitting these five, the variation in average deviation $=$ I I per cent and maximum difference $=42$ per cent. It is interesting to note that both the highest and lowest results are from the same city.

\section{CONCLUSION}

The foregoing study of the present standard of turbidity in water analysis has shown it to be inaccurate, the variations from the average amounting in some cases to over 50 per cent. This could be eliminated largely by the simple expedient of having all these standards prepared by the Bureau of Standards, without changing the specifications at all. But if the standards were thus centrally prepared, it would not be necessary to use the platinumwire method for regulating the size of the particles. Simplicity could be sacrificed for precision in preparing the standard. This would require no change in the routine laboratory comparison of the unknown with the standard. Regardless of the questions of convenience and range, there seems to be no reason why. the standard should not be made precise to at least ro per cent.

There is a possibility that standard samples of dry powdered silica can be prepared sufficiently uniform in size of particle to use directly. In this case certified samples can be furnished the water laboratories, where it would be necessary only to suspend the appropriate amount in distilled water. This question is now being investigated.

The constant advice and encouragement of W. F. Wells, who first brought the problem of turbidity standardization to the attention of the author; the assistance of Prof. E. B. Phelps in furnishing a list of representative water laboratories; and the cooperation of the water laboratories in furnishing samples of their standard turbidity and in giving their opinions respecting the standard, are acknowledged.

Washington, April 25, I9I9. 